\title{
AUTMEDICAÇÃO: UM PROBLEMA TAMBÉM NA HOMEOPATIA.
}

\section{SELF MEDICATION: A PROBLEM IN THE HOMEOPTHY TOO.}

\author{
BOURSCHEID, Andrea ${ }^{1}$; MIGUEL, Marilis D.2; COSTA, Camila K. ${ }^{2}$; ZANIN, Sandra Maria \\ W. ${ }^{2}$; BARREIRA, Sandra Mara W. ${ }^{2}$ \\ 1-Aluna do Curso de Especialização em Atenção Farmacêutica da UFPR; \\ 2-Professora do departamento de farmácia da UFPR; \\ e-mail andreafarma@bol.com.br
}

\begin{abstract}
RESUMO:
A homeopatia é um método terapêutico baseado na lei natural de cura que visa à individualização dos sintomas e tem o objetivo de estimular a reação orgânica no sentido da cura. Por oferecer muitos benefícios à saúde, a homeopatia constitui uma das formas de tratamento que mais se expandem no mundo. Consequentemente, esta realidade vem promovendo um aumento significativo no consumo de medicamentos homeopáticos. Com o objetivo de avaliar 0 conhecimento dos usuários de medicamentos sobre o tratamento homeopático e identificar fatores interferentes neste tratamento, foi realizada uma pesquisa em farmácias homeopáticas de Curitiba-PR utilizando um instrumento de avaliação composto de 20 questões diretas formuladas de acordo com a literatura revisada. Antes de responderem às questões, os usuários de medicamentos homeopáticos leram e assinaram um Termo de Consentimento Livre e Esclarecido. O resultado da pesquisa demonstrou um problema que já é comum na alopatia: a automedicação. Assim, a prática da atenção farmacêutica é indispensável também na homeopatia, pois a falta de informação sobre este método terapêutico considerado inofensivo por muitos, pode comprometer a saúde de quem utiliza estes medicamentos sem prescrição médica.
\end{abstract}

Palavras-chave: atenção farmacêutica, homeopatia.

\begin{abstract}
:
Homeopathy is a therapeutic method based on the natural law of cure that aims the symptoms individualization and has the objective of stimulate the organic reaction towards the cure. Because it offers many benefits to health, homeopathy is one of the treatment forms that most expand in the world. Therefore, this fact promotes a significant enlargement on the homeopathic medicine consumption. With the objective to evaluate the knowledge about the medicine users on the homeopathic treatment and identify some factors which interfere on this treatment, an investigation was developed in homeopathic pharmacies in Curitiba-PR using an evaluation composed by 20 direct questions formulated in accordance with the revised literature. The patients have read and signed a Term of Free and Clarified Assent before answering the questions. The result of this research showed a problem that already is common in the alopathy: the self medication. Thus, the pharmaceutical care practice is indispensable also in the homeopathy, considering that the lack of information on this therapeutic method considered harmless for many people, may compromise the health of whom are users of these medicines without medical prescription.

Keywords: pharmaceutical care, homeopathy.
\end{abstract}

\section{INTRODUÇÃO}

Pelas mãos de Christian Friedrich Samuel Hahnemann, em 1796, a homeopatia iniciouse como método terapêutico baseado na similitude e observação clínica que usa a individualização dos sintomas como sua principal fonte de conhecimento. Através de princípios próprios, a homeopatia avalia e cuida da saúde do homem e também dos animais. Possui 
uma filosofia onde o conceito de saúde é amplo, as enfermidades são profundas e a cura envolve a melhora de todos os sintomas do paciente. É baseada na experiência e medida pelos resultados onde reside sua principal força. Médicos não homeopatas e cientistas das mais variadas áreas têm demonstrado mais interesse em saber como a homeopatia funciona e o que ela tem a oferecer aos pacientes (ROSENBAUM, 2005).

A homeopatia utiliza em sua terapêutica drogas naturais de origem vegetal, mineral e animal. Porém, neste caso o natural não quer dizer isento de efeitos adversos. O medicamento homeopático age por estímulo ao processo natural de cura do organismo e embora seja seguro quando utilizado sob supervisão médica, eles apresentam efeitos adversos como qualquer outro medicamento. Porém, assim como na alopatia, a automedicação é um ato perigoso também na homeopatia e muitas vezes ineficaz. (KOSSAK-ROMANACH, 1984; ROSENBAUM, 2005).

A responsabilidade pelos medicamentos homeopáticos recaiu sobre o farmacêutico que deve dominar aspectos técnicos e promover a atenção farmacêutica. Com isso, os componentes técnicos e humanísticos se harmonizam em torno do paciente com o objetivo de garantir um tratamento efetivo e seguro. (VITHOULKAS, 1980; FONTES, 2001).

A homeopatia vem sendo adotada cada vez mais por médicos e pacientes. Diante desta realidade, qual a compreensão sobre este método terapêutico pelo usuário de medicamentos? A automedicação é considerada uma prática segura por este usuário? Há conhecimento sobre os fatores que podem interferir no tratamento? Por meio da referida pesquisa buscou-se detectar problemas relacionados com medicamentos homeopáticos visando qualificar a dispensação destes medicamentos.

\section{MATERIAL E MÉTODOS}

Para o presente trabalho, foi realizada uma pesquisa com usuários de medicamentos em farmácias homeopáticas do Município de Curitiba no Estado do Paraná.

Foi elaborado um instrumento de avaliação composto de 20 questões diretas formuladas de acordo com a literatura revisada.

O projeto de pesquisa foi submetido ao CEP (Comitê de Ética em Pesquisa) da Universidade Federal do Paraná e a pesquisa foi realizada no período de setembro de 2008 a dezembro de 2008.

\section{RESULTADOS E DISCUSSÃO}

Com base na pesquisa realizada, constatou-se que pessoas do sexo feminino com idade acima de 40 anos são as que mais utilizam medicamentos homeopáticos. O Gráfico 1, apresenta a relação proporcional entre pessoas do sexo masculino e feminino que utilizam medicamentos homeopáticos e o Gráfico 2, apresenta a faixa etária das pessoas que utilizam estes medicamentos.
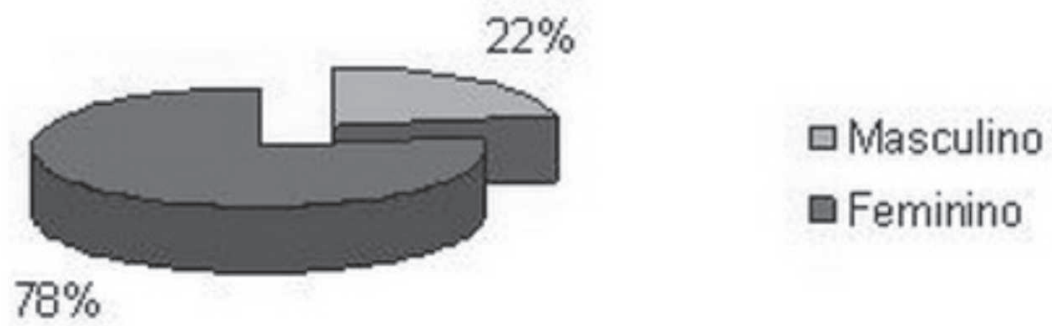

$78 \%$

Gráfico 1 - Distribuição conforme o sexo. 


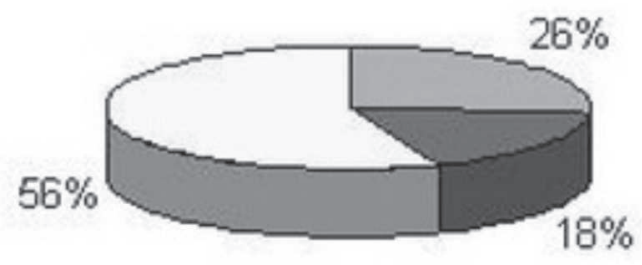

Gráfico 2 - Distribuição conforme a idade.

Entre os usuários participantes da pesquisa, 52\% ficaram sabendo sobre o tratamento homeopático através de médicos, $42 \%$ por amigos e parentes, $12 \%$ através de farmacêuticos e $2 \%$ através de jornais e revistas. A Tabela 1 expressa os motivos pelos quais os usuários buscaram o tratamento homeopático, onde a resposta que prevaleceu foi por ser um tratamento natural com $72 \%$ seguida da resposta por não ter efeitos adversos com $40 \%$.

Tabela 1 - Distribuição dos motivos.

\begin{tabular}{lc}
\hline \multicolumn{1}{c}{ Motivos } & $\%$ \\
\hline Por ser um tratamento natural & $72 \%$ \\
Por não ter efeitos adversos & $40 \%$ \\
Por não estar melhorando com outros tratamentos & $36 \%$ \\
Por ser mais bem atendido nas consultas & $10 \%$ \\
Por oferecer medicamentos mais baratos & $8 \%$ \\
Por ter reações adversas a outros medicamentos & $4 \%$ \\
\hline
\end{tabular}

$\mathrm{Na}$ Tabela 2, podem ser observados os hábitos dos usuários de medicamentos homeopáticos onde o hábito de tomar café predominou com $68 \%$.

Tabela 2 - Distribuição dos hábitos.

\begin{tabular}{ll}
\hline \multicolumn{1}{c}{ Hábitos } & $\%$ \\
\hline Tomar café & $68 \%$ \\
Tomar chá & $52 \%$ \\
Praticar exercícios físicos & $46 \%$ \\
Ingerir bebidas alcoólicas & $20 \%$ \\
Fumar & $10 \%$ \\
\hline
\end{tabular}

Do total de usuários que responderam o instrumento de avaliação, $12 \%$ eram sensíveis à lactose e $52 \%$ apresentavam alguma doença crônica sendo que entre eles $34 \%$ tinham rinite, $14 \%$ hipertensão, $6 \%$ depressão, $2 \%$ diabetes. Dos $36 \%$ de usuários que seguiam outros tratamentos, $18 \%$ faziam tratamento odontológico, $8 \%$ dermatológico, $8 \%$ faziam acupuntura, $6 \%$ utilizavam florais, $6 \%$ faziam fisioterapia, $4 \%$ psicoterapia e $4 \%$ utilizavam fitoterápicos.

Apenas $8 \%$ dos usuários costumavam usar produtos a base de cânfora ou com odores fortes. 
Como pode ser observado no Gráfico 3, dos medicamentos homeopáticos utilizados pelos usuários participantes da pesquisa, prevaleceu com $72 \%$ os que foram indicados por médicos.

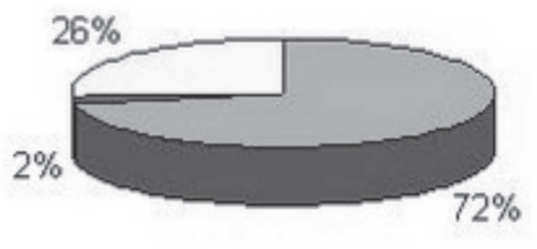

\author{
口Médico \\ Dentista \\ $\square$ Amigo e /ou \\ parente
}

Gráfico 3 - Distribuição dos indicadores dos medicamentos.

Conforme o Gráfico 4, do total de usuários que participaram da pesquisa, 74\% estavam dando continuidade ao tratamento.

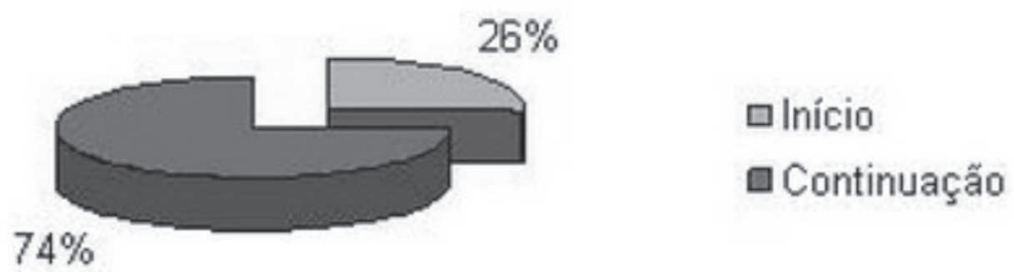

Gráfico 4 - Distribuição da fase de tratamento.

Dos $74 \%$ de usuários que estavam dando continuidade ao tratamento homeopático, $100 \%$ estavam se sentindo melhor; $62,2 \%$ indicavam o medicamento que estavam utilizando para outras pessoas; $8,1 \%$ repetiam a receita por conta própria e 10,8\% suspendiam a medicação por conta própria.

Marcantemente entre estes usuários $91,9 \%$ costumavam conferir o rótulo do medicamento com a receita.

Verifica-se na Tabela 3 que do total de usuários que estavam dando continuidade ao tratamento, predominou com $67,5 \%$ os que ligavam para o médico em caso de dúvida.

Tabela 3 - Distribuição das atitudes em caso de dúvida.

\begin{tabular}{lc}
\hline \multicolumn{1}{c}{ Atitudes em caso de dúvida } & $\%$ \\
\hline Ligavam para o médico & $67,5 \%$ \\
Falavam com o farmacêutico & $13,5 \%$ \\
Aguardavam a próxima consulta & $24,3 \%$ \\
\hline
\end{tabular}


As formas farmacêuticas utilizadas pelos usuários que continuavam o tratamento homeopático podem ser observadas no Gráfico 5 onde os que utilizavam concomitantemente gotas e dose única predominaram com $37 \%$.

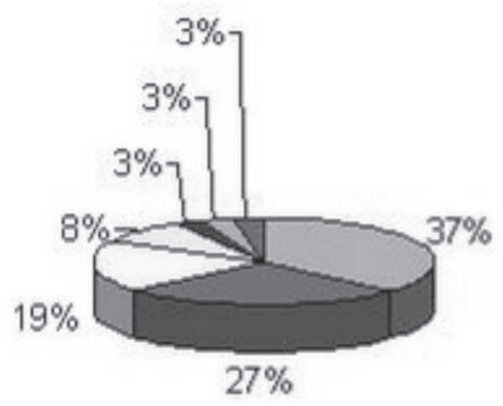

$\square$ Gotas e dose

única

a Gotas

口Dose única

$\square$ Gotas, dose única

e glóbulos

- Gotas e glóbulos

Gotas e

comprimidos

Dose única e

comprimidos

Gráfico 5 - Distribuição das formas farmacêuticas utilizadas.

De acordo com a forma de utilização dos medicamentos homeopáticos pelos usuários, predominantemente $83,8 \%$ destes agitavam o frasco do medicamento na forma líquida antes de utilizar, conforme pode ser observado na Tabela 4.

Tabela 4 - Distribuição das formas de utilização dos medicamentos homeopáticos.

Tabela 4 - Distribuição das formas de utilização dos medicamentos homeopáticos.

\begin{tabular}{lc}
\hline \multicolumn{1}{c}{ Formas de utilização } & $\%$ \\
\hline Agitavam o frasco antes de utilizar (líquido) & $83,8 \%$ \\
Utilizavam em jejum & $43,2 \%$ \\
Utilizavam perto das refeições & $29,7 \%$ \\
Diluíam em água (líquido) & $10,8 \%$ \\
Deixavam dissolver na boca (sólido) & $10,8 \%$ \\
Retiravam do frasco e colocavam na mão (sólido) & $10,8 \%$ \\
Engoliam inteiro (sólido) & $8,1 \%$ \\
Colocavam o frasco direto na boca (sólido) & $5,4 \%$ \\
Mastigavam (sólido) & $2,7 \%$ \\
\hline
\end{tabular}

Dos $67 \%$ de usuários que utilizavam medicamentos em dose única, os que tomavam em jejum prevaleceram com $48 \%$ como pode ser verificado no Gráfico 6. 

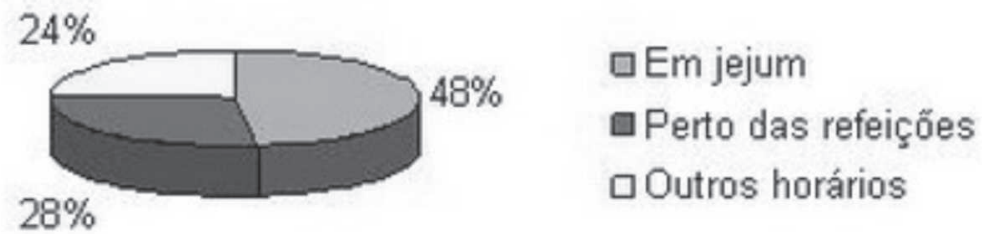

Gráfico 6 - Distribuição do período de utilização dos medicamentos em dose única.

Pela Tabela 5, pode-se observar que entre as dificuldades encontradas na utilização de medicamentos homeopáticos pelos usuários, a dificuldade com os horários foi a mais freqüente com $16,2 \%$.

Tabela 5 - Distribuição das dificuldades encontradas na utilização de medicamentos.

\begin{tabular}{lc}
\hline \multicolumn{1}{c}{ Dificuldades } & $\%$ \\
\hline Em seguir os horários para utilizar & $16,2 \%$ \\
De lembrar de utilizar & $8,1 \%$ \\
Em saber por quanto tempo deveriam utilizar & $2,7 \%$ \\
Em diferenciar mais de um medicamento & $2,7 \%$ \\
Em utilizar o medicamento na seqüência de doses & $2,7 \%$ \\
\hline
\end{tabular}

Quanto à conservação dos medicamentos homeopáticos pelos usuários que responderam esta questão, observa-se na Tabela 6 que a quantidade de usuários que guardavam o medicamento próximo de aparelhos que emitiam radiação prevaleceu com $6 \%$.

Tabela 6 - Distribuição das formas de conservação dos medicamentos.

\begin{tabular}{cc}
\hline Formas de conservação & $\%$ \\
\hline Próximo de aparelhos que emitiam radiação & $6 \%$ \\
Em locais que continham excesso de luz e calor & $2 \%$ \\
\hline
\end{tabular}

\section{CONCLUSÃO}

O perfil dos usuários de medicamentos homeopáticos participantes da pesquisa foi representado como sendo a maioria formada por mulheres acima de 40 anos. Segundo BENEZ (1999), geralmente as mulheres apresentam um estado de enfermidade crônica necessitando de consultas periódicas para avaliações do estado geral, apesar da cura de alguns sintomas locais.

A pesquisa mostrou que na mídia são escassas as referências à homeopatia. Para ROSENBAUM (2005), a explicação para a baixa visibilidade da homeopatia é a omissão crônica do seu verdadeiro alcance.

Diante das informações levantadas observou-se que na homeopatia também há uma tendência à automedicação. Isso decorre da visão distorcida de que a homeopatia é um tratamento inofensivo à saúde. A falta de conhecimento sobre os efeitos que o medicamento 
homeopático não prescrito pode provocar no organismo é o principal motivo que leva a automedicação e ao aumento da freqüência com que as pessoas indicavam seus próprios medicamentos a outras pessoas. Com isso, torna-se clara a necessidade da intervenção do farmacêutico diante deste problema. $O$ paciente deve ser orientado que a mesma doença nem sempre é tratada com o mesmo medicamento e que sem a avaliação do seu estado geral pelo médico homeopata, o medicamento pode causar sintomas que podem comprometer a sua saúde.

Foram identificados fatores que poderiam comprometer o tratamento homeopático como alguns hábitos dos usuários, a utilização e conservação dos medicamentos, a prática de outras terapias e doenças que necessitam ser tratadas com outros medicamentos. Mais da metade dos usuários apresentou alguma doença crônica, tornando-se indispensável o acompanhamento feito pelo farmacêutico.

Houve maior dificuldade com horários nos casos em que os usuários utilizaram mais de uma forma farmacêutica. Nenhum dos usuários sensíveis à lactose utilizou comprimido, assim como nenhum dos usuários diabéticos utilizou glóbulos. Este resultado refletiu a preocupação do médico homeopata em levar em consideração não somente todos os sintomas, mas também todas as doenças apresentadas pelo paciente e nem sempre relatadas por este.

Refletindo diante dos resultados, constatou-se a importância da atenção farmacêutica na homeopatia e a necessidade do farmacêutico ter uma postura frente aos problemas detectados decorrentes da falta de informação sobre o tratamento homeopático.

\section{REFERÊNCIAS}

ASSOCIAÇÃO BRASILEIRA DE FARMACÊUTICOS HOMEOPATAS (ABFH). Manual de Normas Técnicas para Farmácia Homeopática. 3. ㄹ ed. Curitiba: Tempo Integral, 2003, cap. 5 p. $5-7$, cap. 9 p. 1 , cap. 10 p. $2,4,5,9,12-14,16,18,19,22$, cap. 11 p. $1-5$.

\section{ASSOCIAÇÃO BRASILEIRA DE FARMACÊUTICOS HOMEOPATAS (ABFH). Manual do Consumidor de Homeopatia.}

ATTENBOROUGH, A. et al. Dicionário de Medicina Natural. 1. a ed. Rio de Janeiro: Reader's Digest Brasil, 1997, p. 38 - 41, 48, 178, 280.

BAROLLO, C. R. Aos que se tratam pela homeopatia. 3. ${ }^{a}$ ed. São Paulo: Gráfica, 1988, p. $52,58,59$.

BARROS DA SILVA, J. Farmacotécnica Homeopática Simplificada. 2. ${ }^{\text {a }}$ ed. São Paulo: Robe, 1997, p. 157,158.

BENEZ, S. M. Homeopatia 100 Segredos aos que se tratam. São Paulo: Robe, 1999, p. $79,94,111,128$ - 132, 139, 140, 142, 155 - 157, 164, 165, 167, 168, 174 - 176.

BISSON, M. P. Farmácia Clínica e Atenção Farmacêutica. 2. ㄹ ed. Barueri (SP): Manole, 2007, p. $3-6,8,11,13,25,26,35,139$.

BRANDÃO, A. Homeopatia: farmacêuticos discutem serviços, atuação no SUS, insumos, farmacovigilância. Revista Pharmácia Brasileira. Brasília (DF), ano X n.ำ58, p. 43, março/ abril 2007. 
BRANDÃO, A. Entrevista com Dra. Márcia Gutierrez, Presidente da ABFH, Conselho Federal de Farmácia (CFF), 2008. Disponível em:http://sites.mpc.com.br Acesso em: 03/06/ 2008.

BRASIL. Agência Nacional de Vigilância Sanitária. Resolução RDC 67 de 8 de outubro de 2007. Aprova o Regulamento Técnico sobre Boas Práticas de Manipulação de Medicamentos em Farmácias. Anexo I e V.

BRASIL. Ministério da Saúde. Farmacopéia Homeopática Brasileira. 2. a ed. Parte II, São Paulo: Atheneu, 2002, cap. XII p. $22-31$.

CAETANO, N. BPR Guia de Remédios. 8. a ed. São Paulo: Escala, 2006/2007, p. 13 - 15.

CAIRO, N. Guia de Medicina Homeopática. 21. ㄹ ed. São Paulo: Teixeira, 1979, p. 20, 26.

DIAS, A. F. Fundamentos da Homeopatia. Rio de Janeiro: Cultura Médica, 2001, p. 24, 25, $437-439,445-451$.

DUPRAT, H. A Teoria e a Técnica da Homeopatia. 1974, p. 32, 33, 63 - 65.

FONTES, O. L. Farmácia Homeopática Teoria e Prática. 1. a ed. Barueri (SP): Manole, 2001, p. 3 - 11,13,15 - 17, 26 - 33, 36, 41, 42, 44, 45, 48 - 53, 55, 86 - 88, 113, 294.

FRANCHI, S. M. Avaliação da ação antibacteriana dos veículos alcoólicos e aquosos em medicação homeopática na forma líquida. Curitiba, 1996. 37 f. Monografia (Especialização em Ciências Farmacêuticas) - Setor de Ciência da Saúde, Universidade Federal do Paraná.

HAHNEMANN, S. Organon da Arte de Curar. 6. ed. São Paulo: Robe,1996, p. 115, 242.

KOSSAK-ROMANACH, A. Homeopatia em 1000 Conceitos. São Paulo: Eclid, 1984, p. 31, $53,65,70,71,131,134,136,137,158,162,169,171-175,181,340,344,345,362,363$, $367,376,391,409,412,414,415,517,546,573$.

LACERDA, P. Homeopatia em Alergias. São Paulo: Andrei, 1993, p. 46 - 48.

MORAES, O. A Homeopatia Sem Segredos. 2. ${ }^{a}$ ed. Rio de Janeiro: Record, 1987, p. 21 , $24,31,32,37,50,52-54$.

NASSIF, M. R. G. Compêndio de Homeopatia. São Paulo: Robe, 1995, p. 38, 127,128,130 $-133,135,136,267-272$.

ROSENBAUM, P. Homeopatia Medicina Sob Medida. São Paulo: Publifolha, 2005, p. 12, $15-19,29-31,33,50,51,53-55,58,59,63-65,71,75,79,85-87,90,91-95,117-$ 123, 142.

SHARMA, C. H. Manual de Homeopatia e Medicina Natural. São Paulo: Cultrix, 1985, p. $12,15,20,21,63,64,66-68,164$. 
SOARES, A. A. D. Farmácia Homeopática. São Paulo: Andrei, 1997, p. 9, 10, 180, 181.

VITHOULKAS, G. Homeopatia Ciência e Cura. São Paulo: Círculo do Livro, 1980, p. 23, $24,37,39,40,51,53-55,59,60,93,94,154-157,179,180,207,225,317,359,361$ 363.

WEINER, M.; GOSS, K. O Livro Completo de Homeopatia. Rio de Janeiro: Record, 1994, p. $105-108,116,135-137,145,147$.

WELLS, H. Homeopatia para Crianças. $1 .^{a}$ ed. São Paulo: Santos, 1997, p. 13 - 15, 20 , $21,24,30,31$. 